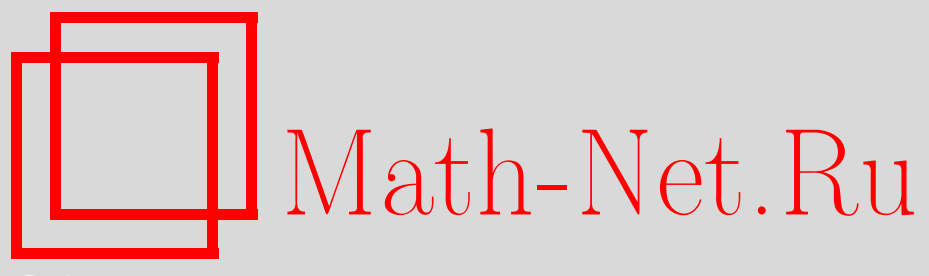

Ю. А. Кордюков, А. А. Яковлев, Задача о числе целых точек в семействах анизотропно расширяющихся областей и ее приложения в спектральной теории, Матем. заметки, 2012, том 92, выпуск 4, 632-635

DOI: https://doi.org/10.4213/mzm9890

Использование Общероссийского математического портала Math-Net.Ru подразумевает, что вы прочитали и согласны с пользовательским соглашением http://www . mathnet.ru/rus/agreement

Параметры загрузки:

IP: 18.234 .197 .8

26 апреля 2023 г., 18:09:56

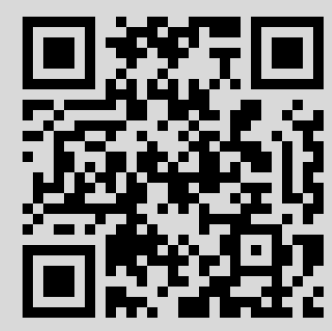




\section{Задача о числе целых точек \\ в семействах анизотропно расширяющихся областей \\ и ее приложения в спектральной теории}

\section{Ю. А. Кордюков, А. А. Яковлев}

1. Постановка задачи и основные результаты. Данная работа посвящена задаче о числе целых точек в семействах анизотропно расширяющихся областей и ее приложениям в спектральной теории дифференциальных операторов.

Пусть $F$ - $p$-мерное линейное подпространство в $\mathbb{R}^{n}$ и $H-q$-мерное ортогональное дополнение к $F$ относительно стандартного скалярного произведения $(\cdot, \cdot)$ в $\mathbb{R}^{n}, p+q=n$. Для любого $\varepsilon>0$ рассмотрим линейное преобразование $T_{\varepsilon}: \mathbb{R}^{n} \rightarrow \mathbb{R}^{n}$ такое, что

$$
T_{\varepsilon}(x)=x, \quad \text { если } \quad x \in F, \quad \text { и } \quad T_{\varepsilon}(x)=\varepsilon^{-1} x, \quad \text { если } \quad x \in H .
$$

Для любого ограниченного множества $S$ в $\mathbb{R}^{n}$ положим

$$
n_{\varepsilon}(S)=\#\left(T_{\varepsilon}(S) \cap \mathbb{Z}^{n}\right), \quad \varepsilon>0 .
$$

Рассмотрим свободную абелеву группу $\Gamma=\mathbb{Z}^{n} \cap F$ ранга $r \leqslant p$. Пусть $V-r$-мерное подпространство $\mathbb{R}^{n}$, порожденное элементами группы $Г$. Группа $\Gamma$ является решеткой в $V$. Обозначим через $\Gamma^{*}$ решетку в пространстве $V$, двойственную к $\Gamma: \Gamma^{*}=\left\{\gamma^{*} \in V\right.$ : $\left.\left(\gamma^{*}, \Gamma\right) \subset \mathbb{Z}\right\}$.

Для любого $x \in V$ через $P_{x}$ обозначим $(n-r)$-мерное аффинное подпространство $\mathbb{R}^{n}$, проходящее через точку $x$ перпендикулярно $V$.

Теорема 1. Для любого подмножества $S$ в $\mathbb{R}^{n}$ такого, что для любого $\gamma^{*} \in \Gamma^{*}$ множество $P_{\gamma^{*}} \cap S$ является ограниченным открытым подмножеством в $P_{\gamma^{*}}$, измеримым по Жордану, имеет место формула

$$
n_{\varepsilon}(S) \sim \frac{\varepsilon^{-q}}{\operatorname{vol}(V / \Gamma)} \sum_{\gamma^{*} \in \Gamma^{*}} \operatorname{vol}_{n-r}\left(P_{\gamma^{*}} \cap S\right), \quad \varepsilon \rightarrow 0 .
$$

Здесь при $r=0$ группы $\Gamma$ и $\Gamma^{*}$ тривиальны, и мы полагаем $\operatorname{vol}(V / \Gamma)=1$.

Исследование асимптотического поведения $n_{\varepsilon}(S)$ при $\varepsilon \rightarrow 0$ для областей общего вида в $\mathbb{R}^{n}$ было начато в работе [1]. В работе [1] доказаны асимптотические формулы для $n_{\varepsilon}(S)$ с нетривиальной оценкой остатка в следующих двух случаях:

1) $S$ - ограниченная открытая область с гладкой границей;

2) $P_{\gamma^{*}} \cap S$ является ограниченной выпуклой областью в $P_{\gamma^{*}}$ для любого $\gamma^{*} \in \Gamma^{*}$.

В несколько иной постановке задача о подсчете числа целых точек в семействе анизотропно расширяющихся областей достаточно подробно изучалась в работах [2], [3] (см. также приведенные в них ссылки и введение в [1]).

В этой работе мы продолжаем изучение остатка в формуле (1), задаваемого формулой

$$
R_{\varepsilon}(S)=n_{\varepsilon}(S)-\frac{\varepsilon^{-q}}{\operatorname{vol}(V / \Gamma)} \sum_{\gamma^{*} \in \Gamma^{*}} \operatorname{vol}_{n-r}\left(P_{\gamma^{*}} \cap S\right) .
$$

Прежде всего, мы доказываем более точные оценки остатка $R_{\varepsilon}(S)$ при дополнительных предположениях на $S$.

Работа выполнена при поддержке Российского фонда фундаментальных исследований (гранты №№ 09-01-00389, 12-01-00519). 
Tеорема 2. Пусть $S$ - такое подмножсество в $\mathbb{R}^{n}$, что для любого $\gamma^{*} \in \Gamma^{*}$ пересечение $P_{\gamma^{*}} \cap S$ является ограниченным открытым множеством в $P_{\gamma^{*}}$ с гладкой гранищей. Тогда выполнены следующие утверждения:

(1) если для любых $\gamma^{*} \in \Gamma^{*} u x \in F \cap V^{\perp}$ пересечение $S \cap\left\{\gamma^{*}+x+H\right\}$ строго выпукло, то имеет место формула

$$
R_{\varepsilon}(S)=O\left(\varepsilon^{2 q /(q+1+2(p-r))-q}\right), \quad \varepsilon \rightarrow 0 ;
$$

(2) если для любого $\gamma^{*} \in \Gamma^{*}$ пересечение $P_{\gamma^{*}} \cap S$ строго выпукло, то

$$
R_{\varepsilon}(S)=O\left(\varepsilon^{2 q /(n-r+1)-q}\right), \quad \varepsilon \rightarrow 0 .
$$

В работах [4], [5] Рэндол предложил рассматривать вместо остатка в аналогичной формуле для области $S$ в случае семейства гомотетичных областей в $\mathbb{R}^{n}$ его средние, взятые по образам области $S$ при вращениях или при вращениях и переносах. Он заметил, что усредненные оценки могут быть существенно лучше, чем индивидуальные оценки. В [4], [5] результаты такого рода были доказаны для ограниченных выпуклых областей с аналитической границей. Результаты Рэндола были распространены Варченко [6] на произвольные ограниченные области с гладкой границей, что высказывалось ранее Арнольдом в качестве гипотезы. Наконец, в [7] усредненные оценки остатка были доказаны для ограниченной области $S$ в случае, если она выпукла, либо если ее граница класса $C^{3 / 2}$. Мы отсылаем читателя к [7], [8] за дальнейшей информацией и ссылками по этой задаче.

В данной работе мы изучаем усредненные оценки остатка $R_{\varepsilon}(S)$, взятые по образам области $S$ при вращениях на элементы некоторой группы ортогональных преобразований евклидова пространства $\mathbb{R}^{n}$. Мы начнем с группы $\mathrm{SO}(H)$ ортогональных преобразований пространства $\mathbb{R}^{n}$, которые оставляют на месте любой вектор из $F$ (и, как следствие, переводят $H$ в себя). Мы будем обозначать через $d h$ меру Хаара на $\mathrm{SO}(n)$ (а также на любой подгруппе в $\mathrm{SO}(n))$.

Теорема 3. Для любого подмножества $S$ в $\mathbb{R}^{n}$ такого, что для любых $\gamma^{*} \in \Gamma^{*} u x \in$ $F \cap V^{\perp}$ пересечение $S \cap\left\{\gamma^{*}+x+H\right\}$ является ограниченным открытым множеством в $P_{\gamma^{*}}$ таким, что либо $S \cap\left\{\gamma^{*}+x+H\right\}$ выпукло, либо граница $S \cap\left\{\gamma^{*}+x+H\right\}$ класса $C^{3 / 2}$, имеет место формула

$$
\int_{S O(H)}\left|R_{\varepsilon}(h S)\right| d h=O\left(\varepsilon^{2 q /(q+1+2(p-r))-q}\right), \quad \varepsilon \rightarrow 0 .
$$

Теперь рассмотрим группу $\mathrm{SO}\left(V^{\perp}\right)$ ортогональных преобразований пространства $\mathbb{R}^{n}$, которые оставляют на месте любой вектор из $V$.

Теорема 4. Для любого подмножества $S$ в $\mathbb{R}^{n}$ такого, что для любого $\gamma^{*} \in \Gamma^{*}$ пересечение $P_{\gamma^{*}} \cap S$ является ограниченным открытым множеством в $P_{\gamma^{*}}$ с гладкой гранищей, имеет место формула

$$
\int_{S O\left(V^{\perp}\right)}\left|R_{\varepsilon}(h S)\right| d h=O\left(\varepsilon^{2 q /(n-r+1)-q}\right), \quad \varepsilon \rightarrow 0 .
$$

Наконец, из теоремы 4 легко следует утверждение о усредненных оценках остатка для всей группы $\mathrm{SO}(n)$.

Теорема 5. Для любого ограниченного открытого множества $S$ в $\mathbb{R}^{n}$ с гладкой гранищей имеет место формула

$$
\int_{S O(n)}\left|R_{\varepsilon}(h S)\right| d h=O\left(\varepsilon^{2 q /(n-r+1)-q}\right), \quad \varepsilon \rightarrow 0 .
$$


2. Приложения к адиабатическим пределам. Как и выше, пусть $F$ - линейное подпространство в $\mathbb{R}^{n}$ и $H=F^{\perp}$. Рассмотрим ассоциированное слоение $\mathcal{F}$ на $n$-мерном торе $\mathbb{T}^{n}=\mathbb{R}^{n} / \mathbb{Z}^{n}$ со слоями

$$
L_{x}=x+F \quad \bmod \mathbb{Z}^{n}, \quad x \in \mathbb{T}^{n} .
$$

Разложение пространства $\mathbb{R}^{n}$ в виде прямой суммы $\mathbb{R}^{n}=F \oplus H$ индуцирует разложение $g=g_{F}+g_{H}$ стандартной евклидовой метрики $g$ на $\mathbb{T}^{n}$ в виде суммы касательной и трансверсальной составляющих. Определим семейство $g_{\varepsilon}$ римановых метрик на $\mathbb{T}^{n}$ по формуле

$$
g_{\varepsilon}=g_{F}+\varepsilon^{-2} g_{H}, \quad \varepsilon>0 .
$$

Для любого $\varepsilon>0$ рассмотрим оператор $H_{\varepsilon}$ в пространстве $C^{\infty}\left(\mathbb{T}^{n}\right)$, определяемый в декартовых координатах $\left(x_{1}, x_{2}, \ldots, x_{n}\right)$ пространства $\mathbb{R}^{n}$ по формуле

$$
H_{\varepsilon}=\sum_{j, \ell=1}^{n} g_{\varepsilon}^{j \ell}\left(\frac{\partial}{\partial x_{j}}-2 \pi i a_{j}\right)\left(\frac{\partial}{\partial x_{\ell}}-2 \pi i a_{\ell}\right),
$$

где $A=\left(a_{1}, \ldots, a_{n}\right) \in \mathbb{R}^{n}, g_{\varepsilon}^{j \ell}$ - элементы матрицы, обратной к матрице метрики $g_{\varepsilon}$. Оператор $H_{\varepsilon}$ можно рассматривать как магнитный оператор Шрёдингера на торе $\mathbb{T}^{n}$, ассоциированный с метрикой $g_{\varepsilon}$ и с постоянным магнитным потенциалом $\mathbf{A}=\sum_{j=1}^{n} a_{j} d x_{j}$. Переход к пределу при $\varepsilon \rightarrow 0$ часто называется переходом $\kappa$ адиабатическому пределу. Это понятие было введено Виттеном в 1985 г. при изучении глобальных аномалий в теории струн (см. [9] по поводу исторических замечаний и ссылок).

Легко видеть [1], что функция

$$
N_{\varepsilon}(\lambda):=\sharp\left\{k \in \mathbb{N}: \lambda_{k}(\varepsilon)<\lambda\right\}
$$

распределения собственных значений $\left\{\lambda_{k}(\varepsilon): k \in \mathbb{N}\right\}$ оператора $H_{\varepsilon}$ связана с числом целых точек в эллипсоиде $T_{\varepsilon}\left(B_{\sqrt{\lambda}}(A)\right)$ по формуле

$$
N_{\varepsilon}\left(4 \pi^{2} \lambda\right)=n_{\varepsilon}\left(B_{\sqrt{\lambda}}(A)\right), \quad \lambda \in \mathbb{R},
$$

где $B_{\sqrt{\lambda}}(A)$ - шар с центром в точке $A$ радиуса $\sqrt{\lambda}$. В качестве непосредственного следствия теоремы 2 получается следующая асимптотическая формула для функции распределения собственных значений, которая улучшает формулу, полученную в [1].

Теорема 6. Для любого $\lambda>0$ справедлива следующая асимптотическая формула при $\varepsilon \rightarrow 0$ :

$$
N_{\varepsilon}(\lambda)=\varepsilon^{-q} \frac{\omega_{n-r}}{\operatorname{vol}(V / \Gamma)} \sum_{\gamma^{*} \in \Gamma^{*}}\left(\frac{\lambda}{4 \pi^{2}}-\left|\gamma^{*}-A\right|^{2}\right)^{(n-r) / 2}+O\left(\varepsilon^{2 q /(n-r+1)-q}\right),
$$

где $\omega_{n-r}$ - обвем шара единичного радиуса в пространстве $\mathbb{R}^{n-r}$.

\section{СПИСОК ЦИТИРОВАННОЙ ЛИТЕРАТУРЫ}

[1] Ю.А. Кордюков, А.А. Яковлев, Алгебра и анализ, 23:6 (2011), 80-95. [2] N. A. Nikichine, M. M. Skriganov, C. R. Acad. Sci. Paris Sér. I Math., 321:6 (1995), 671-675. [3] Н.А. Никишин, М. М. Скриганов, Алгебра и анализ, 10:1 (1998), 68-87. [4] B. Randol, Trans. Amer. Math. Soc., 139 (1969), 271-278. [5] B. Randol, Trans. Amer. Math. Soc., 139 (1969), 279-285. [6] А.Н. Варченко, Функи. анализ и его прил., 17:2 (1983), 1-6. [7] L. Brandolini, S. Hofmann, A. Iosevich, Geom. Funct. Anal., 13:4 
(2003), 671-680. [8] П. М. Грубер, К. Г. Леккеркеркер, Геометрия чисел, Наука, М., 2008. [9] Yu. A. Kordyukov, A. A. Yakovlev, $C^{*}$-Algebras and Elliptic Theory. II, Trends Math., Birkhäuser-Verlag, Basel, 2008, 123-144.

Ю. А. Кордюков

Поступило

Институт математики с ВЦ УНЦ РАН, г. Уфа

07.04.2012

E-mail: yurikor@matem.anrb.ru

\section{А. А. Яковлев}

Институт математики с ВЦ УНЦ РАН, г. Уфа

E-mail: yakovlevandrey@yandex.ru 